\title{
Subcarrier allocation in coded OFDMA uplink systems: Diversity versus CFO
}

\author{
Antonia Maria Masucci, Inbar Fijalkow, Elena Veronica Belmega \\ ETIS/ENSEA - Université de Cergy Pontoise - CNRS \\ 6 Avenue de Ponceau, 95014 Cergy, France \\ Email: masucci@ensea.fr, inbar.fijalkow@ensea.fr, belmega@ensea.fr
}

\begin{abstract}
In this paper, we analyze the optimal blockwise subcarrier allocation scheme in coded Orthogonal Frequency Division Multiple Access (OFDMA) uplink systems without channel state information at the transmitter side. Due to the Discrete Fourier Transform, the subcarrier gains in OFDMA systems are correlated and this correlation plays a determinant role since it causes a degradation of the transmission performance. As a consequence, there exist some subcarrier allocation schemes which are preferable to achieve higher diversity gains. We propose the minimization of an inter-carrier sum-correlation function defined as the sum of correlations of each subcarrier with respect to the others in the same allocation scheme. We show the robustness of the proposed blockwise allocation policy to the effect of carrier frequency offsets (CFO). Moreover, we propose the block-size which provides the best trade-off between diversity and CFO for practical values of CFO. Finally, numerical simulations validating our results are presented.
\end{abstract}

\section{INTRODUCTION}

The increasing demand for high transmission data rates has lead to the development of highly adaptive communication systems. Due to its flexibility, OFDMA has been proposed as the downlink transmission scheme for wireless standards of 4G technology (WiMAX, LTE), [1]. Recent works analyze OFDMA uplink transmission with respect to the analysis of frequency mismatches due to the sensitivity to carrier frequency offsets (CFO), [2]. CFO, generated by the frequency misalignments between the mobile users' oscillator and the base station, causes the loss of orthogonality among subcarriers by producing a shift of the received signal causing intercarrierinterference (ICI).

OFDMA uplink systems without CFO and with independent subcarriers achieve channel diversity gain by using any allocation scheme. However, due to the Discrete Fourier Transform, the OFDMA systems undergo correlated subcarriers that imply a degradation of the transmission performance. We are therefore looking at the subcarrier allocation schemes that are likely to obtain an increased diversity gain, when choosing the block-size. CFO increases the loss of diversity due to ICI.

In OFDMA uplink transmission, users spread their information across the set of available subcarriers. Subcarrier allocation techniques are used to split the available bandwidth between the users enabling each user to have a unique access. In the case with no channel state information (CSI) available, the most used allocation schemes are the blockwise and interleaved allocations, [3]. In the blockwise allocation scheme, subsets of adjacent subcarriers are allocated to each user. The interleaved allocation scheme is a special case where subcarriers are uniformly spaced from each other at a distance equal to the number of users (block-size $b$ is equal to one). The interleaved allocation is usually considered to benefit from frequency diversity when no CSI (IEEE 802.16) [4]. However, robustness to CFO should be improved by larger block-sizes.

In the case with full CSI, the authors in [5] propose an optimal block-size as a good balance between the frequency diversity gain and robustness against CFO. A semi-interleaved subcarrier allocation scheme in OFDMA/SC-FDMA systems capable of achieving the diversity gain with minimum $\mathrm{CFO}$ interference has been proposed in [6] restricted to the specific case where each user in the system transmits one symbol spread on all subcarriers. In [7], we study the optimal blocksize allocation schemes for SC-FDMA (Single Carrier FDMA) uplink systems without CFO and without CSI at the transmitter. We look at the effects of correlation on the transmission performance, we provide an analytical expression of the correlation between subcarriers and, analyzing all possible blockwise allocation schemes in function of the block-size, we find the analytical expressions of the optimal blockwise allocation schemes that achieve maximum diversity.

In this work, we analyze the case of uplink coded OFDMA systems with CFO and without CSI. In general, uncoded OFDMA can not exploit the frequency diversity in the channel, therefore the use of channel coding with OFDMA will reduce the errors resulting from the multipath fading environment recovering the diversity gain [8]. In order to enhance the frequency diversity, we propose to minimize the correlation between subcarrier gains similarly to [7]. We define the sum-correlation function as the sum of correlations of each subcarrier with respect to the others in the same allocation scheme and we show the robustness of the proposed blockwise allocation policy to CFO. When the values of CFO increase, because of its negative effects, the smallest block-sizes are no longer optimal as opposed to [7], the case without CFO. We propose a unique optimal block-size that minimizes the sumcorrelation. Numerical Monte Carlo simulations validate this value as the best trade-off between diversity and CFO.

\section{SySTEM MODEL}

We consider an OFDMA uplink system with $N_{u}$ mobile users communicating with a base station (BS) or access point. No CSI is available at the transmitter side. The total 
bandwidth $B$ is divided into $N_{p}$ subcarriers and we denote by $M=N_{p} / N_{u}$ the number of subcarriers per user. We take $N_{p}$ equal to an integer power of two in order to optimize the Discrete Fourier Transform (DFT) processing. Moreover, we assume both the number of users $N_{u}$ and the block-size $b$ to be integer powers of two to have fair (in the sense that the number of allocated subcarriers is the same for all users) allocation schemes among the users and a fully utilized spectrum.

The signal before the DFT at the receiver is given in (1), in the next page, by [5], where $L$ is the length of the cyclic prefix. The vector $\mathbf{h}^{(u)}=\left[h_{0}^{(u)}, \ldots, h_{L-1}^{(u)}\right]$ represents the discrete channel impulse response of user $u$. The elements $a_{k}^{(u)}$ are the symbols at the output of the Inverse DFT (IDTF) given by

$$
\mathbf{a}^{(u)}=\left(\begin{array}{c}
a_{N_{p}-1}^{(u)} \\
\vdots \\
a_{0}^{(u)}
\end{array}\right)=\mathbf{F}^{-1} \boldsymbol{\Pi}_{b}^{(u)} \mathbf{x}^{(u)}
$$

with $\mathbf{F}^{-1}$ the IDFT matrix, $\mathbf{x}^{(u)}$ is the signal transmitted by user $u$ and $\Pi_{b}^{(u)}$ is the $N_{p} \times N_{p}$ subcarrier allocation matrix whose elements are equal to 1 for the carriers allocated to user $u$ and equal to 0 for the carriers allocated to user $v$ with $v \neq u$ according to the considered block-size $b \in \beta=$ $\left\{1,2,4, \ldots, \frac{N_{p}}{N_{u}}\right\}=\left\{2^{0}, 2^{1}, 2^{2}, \ldots, 2^{\log \frac{N_{p}}{N_{u}}}\right\}$. The diagonal elements $\delta_{k}^{(u)}$ are the frequency shift coefficients given by $\delta_{k}^{(u)}=e^{\frac{j 2 \pi k \delta f_{c}^{(u)} T}{N_{p}}}, k \in\left\{0, \ldots, N_{p}+L-1\right\}$, where $\frac{\delta f_{c}^{(u)} T}{N_{p}}$ is the normalized CFO of user $u$.

Discarding in the signal at the input of the receiver DFT, given in (1), the last $L$ components corresponding to the cyclic prefix and rearranging the terms we have:

$\left(\begin{array}{c}y_{N_{p}-1} \\ \vdots \\ y_{0}\end{array}\right)=\sum_{u=1}^{N_{u}} \mathbf{h}_{\text {circ }}^{(u)} \underbrace{\left(\begin{array}{ccc}\delta^{0^{(u)}} & & \\ & \ddots & \\ 0 & & \\ \delta^{\left(N_{p}-1\right)^{(u)}}\end{array}\right)}_{\delta^{(u)}}\left(\begin{array}{c}a_{N_{p}-1}^{(u)} \\ \vdots \\ a_{0}^{(u)}\end{array}\right)+\left(\begin{array}{c}n_{N_{p}-1} \\ \vdots \\ n_{0}\end{array}\right)$

and

$$
\mathbf{h}_{\text {circ }}^{(u)}=\left(\begin{array}{ccccc}
h_{0}^{(u)} & \cdots & h_{L-1}^{(u)} & & 0 \\
& \ddots & & \ddots & \\
& & & & h_{L-1}^{(u)} \\
h_{L-1}^{(u)} & 0 & & & \\
\vdots & \ddots & & \ddots & \\
h_{1}^{(u)} & \cdots & h_{L-1}^{(u)} & & h_{0}^{(u)}
\end{array}\right)
$$

Denoting by $\mathbf{r}=\mathbf{F y}$, the received signal after the DFT

$$
\mathbf{r}=\sum_{u=1}^{N_{u}} \mathbf{H}^{(u)} \boldsymbol{\Delta}^{(u)} \boldsymbol{\Pi}_{b}^{(u)} \mathbf{x}^{(u)}+\hat{\mathbf{n}},
$$

where $\tilde{\mathbf{n}}=\mathbf{F n}$ is the $N_{p} \times 1$ additive Gaussian noise with variance $\sigma_{n}^{2} \mathbf{I}, \mathbf{H}^{(u)}=\mathbf{F} \mathbf{h}_{\text {circ }}^{(u)} \mathbf{F}^{-1}$ is the diagonal channel matrix of user $u$ with the $(k, k)$-entry given by $H_{k}^{(u)}=\sum_{m=0}^{L-1} h_{m}^{(u)} e^{-j 2 \pi m k / N_{p}}$, the $N_{p} \times N_{p}$ matrix
$\boldsymbol{\Delta}^{(u)}=\mathbf{F} \delta^{(u)} \mathbf{F}^{-1}$ represents the loss of orthogonality induced by CFO. In particular, we have the $(l, k)$ element of $\Delta^{(u)}$ :

$$
\begin{aligned}
\Delta_{\ell, k}^{(u)} & =\frac{1}{N_{p}} \sum_{i=0}^{N_{p}-1} e^{j 2 \pi i \delta f / N_{p}} e^{-j 2 \pi i(\ell-k) / N_{p}} \\
& =\frac{1}{N_{p}} \frac{\sin (\pi(\delta f+k-\ell))}{\sin \left(\frac{\pi}{N_{p}}(\delta f+k-\ell)\right)} e^{\pi j\left(1-\frac{1}{N_{p}}\right)(\delta f+k-\ell)} .
\end{aligned}
$$

In the sequel, we denote $\tilde{\mathbf{H}}^{(u)} \triangleq \mathbf{H}^{(u)} \boldsymbol{\Delta}^{(u)}$. Note that in (3) several terms due to cyclic prefix were not taken into account [5], that have almost no effect unless very large cyclic prefix is considered.

\section{Sum-CORRELATION: CASE Without CFO}

In order to optimize the frequency diversity when the system is not affected by CFO, we define a measure of correlation between subcarriers, that we call sum-correlation function. We note that when the system is not affected by CFO the matrix $\boldsymbol{\Delta}^{(u)}$ in (3) is equal to $\mathbf{I}$.

\section{A. Sum-correlation minimization}

Assuming that there is no CFO in the system and the channel impulse responses $h_{m}^{(u)}$ are independent but distributed accordingly to the same complex Gaussian distribution $\operatorname{e\mathcal {N}}\left(0, \sigma_{h}^{2}\right)$, we consider the following sum-correlation function, [7]:

$$
\begin{aligned}
\Gamma_{m}(b) & =\sum_{c_{u} \in \mathfrak{C}_{u}} \mathbb{E}\left[H_{m}^{(u)} H_{c_{u}}^{(u) *}\right] \\
& =\mathbb{E}\left[\left|H_{m}^{(u)}\right|^{2}\right]+\sum_{\substack{c_{u} \in \mathfrak{C}_{u} \\
c_{u} \neq m}} \mathbb{E}\left[H_{m}^{(u)} H_{c_{u}}^{(u) *}\right] \\
& =\frac{\sigma_{h}^{2}}{N_{p}} L+\frac{\sigma_{h}^{2}}{N_{p_{c_{u}} \in \mathcal{C}_{u}}} \sum_{\sin \left[\frac{\pi}{N_{p}}\left(m-c_{u}\right)\right]} \frac{\sin \left[\frac{\pi L}{N_{p}}\left(m-c_{u}\right)\right]}{-\pi j \frac{(L-1)}{N_{p}}\left(m-c_{u}\right)}
\end{aligned}
$$

for all $u \in N_{u}$, where $m \in \mathcal{C}_{u}$ is the reference subcarrier and

$$
\mathcal{C}_{u}=\underset{k \in\left\{1,2,3, \ldots, \frac{N_{p}}{b N_{u}}\right\}}{\bigcup}\left\{(k-1) b N_{u}+(u-1) b+i, \forall i \in\{1, \ldots, b\}\right\} .
$$

The set $\mathcal{C}_{u}$ is composed of all indices of subcarriers allocated to user $u$ given a block-size $b$ allocation scheme.

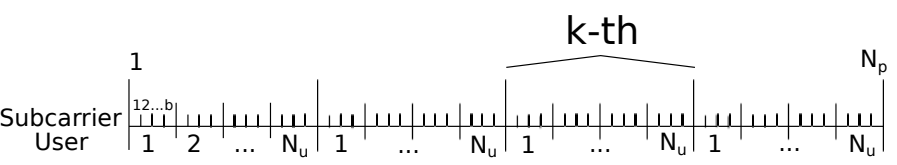

Fig. 1: The total $N_{p}$ subcarriers are divided into $\frac{N_{p}}{b N_{u}}$ large-blocks. The $k$-th large-block contains $b N_{u}$ subcarriers, which are divided into $N_{u}$ block of size $b$, one for each user.

The ratio $\frac{N_{p}}{b N_{u}}$ represents the number of blocks that can be allocated to each user given a block-size $b$. We consider, 


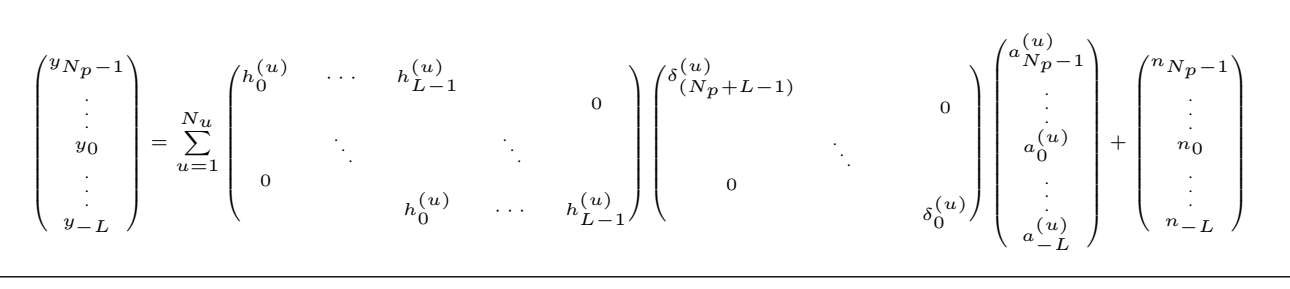

therefore, that the total $N_{p}$ subcarriers are divided into $\frac{N_{p}}{b N_{u}}$ large-blocks that contain $b N_{u}$ subcarriers corresponding to the $N_{u}$ blocks, one for each user, of size $b$. The set $\mathcal{C}_{u}$ is the union of indices of subcarriers allocated to user $u$ in all these largeblocks. Inside of the large-block of index $k$, the indices of the $b$ subcarriers allocated to user $u$ are $(k-1) b N_{u}+(u-1) b+i$, $\forall i \in\{1, \ldots, b\}$, where $(k-1) b N_{u}$ corresponds to the previous $k-1$ large-blocks and $(u-1) b$ corresponds to the previous allocated users $(1,2, \ldots, u-1)$, see Figure 1. The function $\Gamma_{u, m}(b)$ is the sum of the correlations between subcarriers that are in the same allocation scheme.

In the next Theorem, we give the set of optimal block-sizes that minimize the sum-correlation function given in (4).

Theorem 1 ([7]): We consider our uplink system with no CFO and $N_{p}$ subcarriers, $N_{u}$ users and a cyclic prefix of size L. Given $\beta^{*} \triangleq\left\{1,2,4, \ldots, \frac{N_{p}}{N_{u} L}\right\}$, we have the optimal allocation set $\beta^{*}=\underset{b \in \beta}{\arg \min } \Gamma_{m}(b)$ and $\Gamma_{m}\left(b^{*}\right)=\frac{\sigma_{h}^{2}}{N_{u}}$ for any block-size $b^{*} \in \beta^{*}$ and for any reference subcarrier $m \in \mathcal{C}_{u}$.

We observe that the minimum value of the sum-correlation function depends only on the number of users and the variance of the channel impulse response, and that the largest optimal block-size $b_{\max }^{*}=\frac{N_{p}}{N_{u} L}$, is a function of system's parameters: number of subcarriers, number of users and cyclic prefix length.

\section{B. Numerical Validations}

In this section, we present some numerical validations. In particular, we show that $\beta^{*}$ in Theorem 1 minimizes the outage probability. This explains that the outage probability, which is a measure of the reliability of the system [9], increases when the correlation among subcarriers is increasing. The outage probability of the system is defined as the maximum of the outage probabilities of the users in the system $P_{\text {out }}=$ $\max _{u}\left\{P_{\text {out }}^{(u)}\right\}$ where $P_{\text {out }}^{(u)}=\operatorname{Pr}\left\{C_{b}^{(u)}<R^{(u)}\right\}$ with $R^{(u)}$ a fixed spectral efficiency at user $u$ and $C_{b}^{(u)}$ is the user instantaneous achievable spectral efficiency assuming singleuser decoding at the BS [10], given by

$$
C_{b}^{(u)}=\frac{N_{u}}{B} \log _{2}\left|\mathbf{I}+\mathbf{H}^{(u)} \boldsymbol{\Pi}_{b}^{(u)} \mathbf{H}^{(u) \dagger}\left(\mathbf{I} \sigma_{n}^{2}+\sum_{\substack{v=1 \\ v \neq u}}^{N_{u}} \mathbf{H}^{(v)} \boldsymbol{\Pi}_{b}^{(v)} \mathbf{H}^{(v) \dagger}\right)^{-1}\right|
$$

considering the transmitted symbols in (2) distributed accordingly to the gaussian distribution such that $\mathbb{E}\left[\mathbf{x}^{(u)} \mathbf{x}^{(u) H}\right]=\mathbf{I}$

In Figure 2, we evaluate the outage probability $P_{\text {out }}$ for the scenario $N_{u}=4, L=8$ and $R^{(u)}=0.5 \mathrm{bits} / \mathrm{s} / \mathrm{Hz}$. We observe that the optimal block-sizes are the ones that correspond to the outage probabilities which have a higher decreasing speed as a function of the SNR. We have that the curves with $b^{*} \in \beta^{*}=\{1,2\}$ (in this case $b_{\max }^{*}=\frac{64}{4 \times 8}=2$ ) overlap and they represent the lower outage probability, hence the optimal block-sizes.

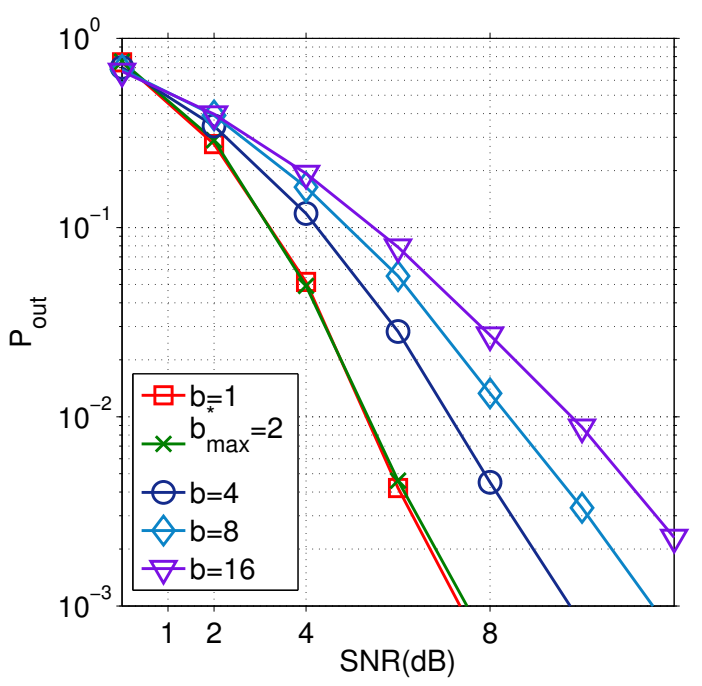

Fig. 2: Outage probability for an OFDMA system without CFO with $N_{p}=64, N_{u}=4$ and $L=8$. The optimal block-sizes are $b^{*} \in \beta^{*}=\{1,2\}$.

\section{ROBUSTNESS TO CFO}

Similarly to the approach in Section III, we extend the definition of the sum-correlation function to the case in which the system undergoes CFO:

$$
\begin{aligned}
\Gamma_{u, m}^{C F O}(b, \delta f) & \triangleq \sum_{c_{u} \in \mathfrak{C}_{u}} \mathbb{E}\left[\tilde{\mathbf{H}}_{m}^{(u)} \tilde{\mathbf{H}}_{c_{u}}^{(u) \dagger}\right] \\
& =\sum_{c_{u} \in \mathfrak{C}_{u}} \sum_{k \in \mathcal{C}_{u}} \mathbb{E}\left[H_{m}^{(u)} H_{c_{u}}^{(u) *}\right] \Delta_{m, k} \Delta_{c_{u}, k}^{*} \\
& =\frac{\sigma_{h}^{2}}{N_{p}^{3}} L \sum_{k \in \mathcal{C}_{u}} \frac{1-\cos (2 \pi(\delta f+k)-2 \pi m)}{1-\cos \left(\frac{2 \pi}{N_{p}}(\delta f+k)-\frac{2 \pi}{N_{p}} m\right)}+ \\
& +\frac{\sigma_{h}^{2}}{N_{p}} \sum_{c_{u} \in \mathfrak{C}_{u}} e^{-\pi j \frac{(L-1)}{N_{p}}\left(m-c_{u}\right)} \frac{\sin \left[\pi \frac{L}{N_{p}}\left(m-c_{u}\right)\right]}{\sin \left[\frac{\pi}{N_{p}}\left(m-c_{u}\right)\right]} \times \\
& \times \frac{1}{N_{p}^{2}} \sum_{k \in \mathfrak{C}_{u}} e^{-\pi j\left(1-\frac{1}{N_{p}}\right)\left(m-c_{u}\right)} \times \\
\times & \frac{\left[\cos \left(\pi\left(m-c_{u}\right)\right)-\cos \left(2 \pi(\delta f+k)-\pi\left(m+c_{u}\right)\right)\right]}{\left[\cos \left(\frac{\pi}{N_{p}}\left(m-c_{u}\right)\right)-\cos \left(\frac{2 \pi}{N_{p}}(\delta f+k)-\frac{\pi}{N_{p}}\left(m+c_{u}\right)\right)\right]}
\end{aligned}
$$


where $m \in \mathcal{C}_{u}$ is the reference subcarrier, $\delta f$ represents the $\mathrm{CFO}$ of user $u$ and $\tilde{\mathbf{H}}_{m}^{(u)}=\left(H_{m}^{(u)} \Delta_{m, 1}, \ldots, H_{m}^{(u)} \Delta_{m, N_{p}}\right)$ represents the $m$-th row of the matrix $\tilde{\mathbf{H}}^{(u)}$.

In the next Proposition, we study the behavior of the CFO sum-correlation function when the CFOs go to zero. We show that this function can be approximated with the sumcorrelation function defined in the case without CFO in (4). This implies that we have robustness to CFO.

Proposition 1: When the CFO coefficient $\delta f$ becomes very small $(\delta f \rightarrow 0)$ the sum-correlation function defined in (6) tends to the sum-correlation function defined in the case without CFO for any block-size b, i.e. $\lim _{\delta f \rightarrow 0} \Gamma_{u, m}^{C F O}(b, \delta f)=\Gamma_{m}(b)$.

Proof: We denote by

$$
\begin{aligned}
& S_{m, c_{u}}(b, \delta f) \triangleq \frac{1}{N_{p}^{2}} \sum_{k \in \mathcal{C}_{u}} e^{-\pi j\left(1-\frac{1}{N_{p}}\right)\left(m-c_{u}\right)} \times \\
& \times \frac{\left[\cos \left(\pi\left(m-c_{u}\right)\right)-\cos \left(2 \pi(\delta f+k)-\pi\left(m+c_{u}\right)\right)\right]}{\left[\cos \left(\frac{\pi}{N_{p}}\left(m-c_{u}\right)\right)-\cos \left(2 \frac{\pi}{N_{p}}(\delta f+k)-\frac{\pi}{N_{p}}\left(m+c_{u}\right)\right)\right]}
\end{aligned}
$$

In what follows, we consider the case $m=c_{u}$ since it represent the predominant term in the sum-correlation function. Then, we have

$$
\begin{aligned}
S_{m, m}(b, \delta f)= & \frac{1}{N_{p}^{2}} \sum_{k \in \mathfrak{C}_{u}} \frac{1-\cos (2 \pi(\delta f+k)-2 \pi m)}{1-\cos \left(\frac{2 \pi}{N_{p}}(\delta f+k)-\frac{2 \pi}{N_{p}} m\right)} \\
& =\frac{1}{N_{p}^{2}} \sum_{k \in \mathfrak{C}_{u}} \frac{1-\cos (2 \pi \delta f)}{1-\cos \left(\frac{2 \pi}{N_{p}} \delta f+\frac{2 \pi}{N_{p}}(k-m)\right)} \\
& =\frac{1}{N_{p}^{2}}\left[\frac{1-\cos (2 \pi \delta f)}{1-\cos \left(\frac{2 \pi}{N_{p}} \delta f\right)}+\right. \\
& \left.+\sum_{k \neq m} \frac{1-\cos (2 \pi \delta f)}{1-\cos \left(\frac{2 \pi}{N_{p}} \delta f+\frac{2 \pi}{N_{p}}(k-m)\right)}\right] . \text { (8) }
\end{aligned}
$$

We observe that when $\delta f$ becomes very small, we have:

$$
\frac{1-\cos (2 \pi \delta f)}{1-\cos \left(\frac{2 \pi}{N_{p}} \delta f\right)} \underset{\delta f \rightarrow 0}{\longrightarrow} N_{p}^{2}
$$

and also:

$$
\sum_{k \neq m} \frac{1-\cos (2 \pi \delta f)}{1-\cos \left(\frac{2 \pi}{N_{p}} \delta f+\frac{2 \pi}{N_{p}}(k-m)\right)} \underset{\delta f \rightarrow 0}{\longrightarrow} 0,
$$

This implies that the $S_{m, m}(b, \delta f)$ in (8) tends to one when $\delta f$ goes to zero:

$$
S_{m, m}(b, \delta f) \underset{\delta f \rightarrow 0}{\longrightarrow} 1 .
$$

This means that when the CFO $\delta f$ goes to zero we can approximate the CFO sum-correlation function with the sumcorrelation function defined in (4).

In practical cases in which the CFOs take moderate values, we propose a unique optimal block-size that achieves diversity: Since CFO yields a diversity loss, in the presence of moderate values of CFO the optimal block-size allocation is $b_{\text {max }}^{*}=N_{p} / N_{u} L$.

This means that, in presence of $\mathrm{CFO}, b_{\max }^{*}$ represents the good trade-off between CFO and diversity. We have found that the optimal blok-sizes that achieve maximum diversity are the ones that minimize the correlation between subcarriers and we know that, in general, in the presence of CFO in the system larger block-sizes are preferable to oppose the effect of CFO. Above, in presence of CFO, we propose the largest optimal block-size $b_{\max }^{*}$ as the block-size that represents the best tradeoff between diversity and CFO: since it is the largest between the block-sizes that achieve diversity it better contrasts the effect of CFO. This observation is validated by extensive numerical simulations one of which is illustrated in the next section.

\section{Numerical VAlidations}

In Figure 3, we plot the outage probability of an OFDMA system with CFO (marker lines) against the outage probability of an OFDMA system without CFO (dashed lines). The CFO for each user is independently uniformly generated in $[0,0.1]$ and $R^{(u)}=1 \mathrm{bits} / \mathrm{s} / \mathrm{Hz}$. We can see that the curves in the CFO case fit very well the outage probability curves without CFO. In particular, they appear in decreasing order of block-size. This validates our analytical analysis on the approximation of the CFO sum-correlation function to the case without CFO when the CFO goes to zero. Moreover, we observe that in the two cases we have the same optimal block-sizes set, given by $\beta^{*}=\{1,2\}$.

In the next two simulation results, we use QPSK modulation, $N_{p}=128$ and $L=32$. We use a concatened coding, which consists in cascading two or more simple codes in a serial manner, where the resulting composite code is equivalent to a much longer code in performance and lower in decoding complexity. In particular, we use the following pair: a Reed Solomon code, $R S(64,48,8)$, as the outer code with a rate$1 / 2$ convolutional coding with constraint length 7 and code generators $(133,171)$ in octal notation as the inner code.

Figure 4 illustrates the Bit Error Rate (BER) curves for an OFDMA system with CFO independently and uniformly generated for each user in $[0,0.01]$. We observe that for these low values of CFO we have the optimal block-sizes given by $\beta^{*}=\{1,2\}$. Thus, a similar observation as in section III can be made. Figure 5 illustrates the BER curves for an OFDMA system with CFO independently and uniformly generated for each user in [0,0.03]. We observe that in this case we have a unique optimal block-size given by $b_{\max }^{*}=2$. This means that when the $\mathrm{CFO}$ values are increasing the optimal compromise between diversity and CFO is represented by the largest blocksize in the set proposed without $\mathrm{CFO}, b_{\max }^{*}$.

Many others simulations, for different values of the parameters, have been performed and similar observations were made. Moreover, more powerful coding schemes guarantee a gain more important. 


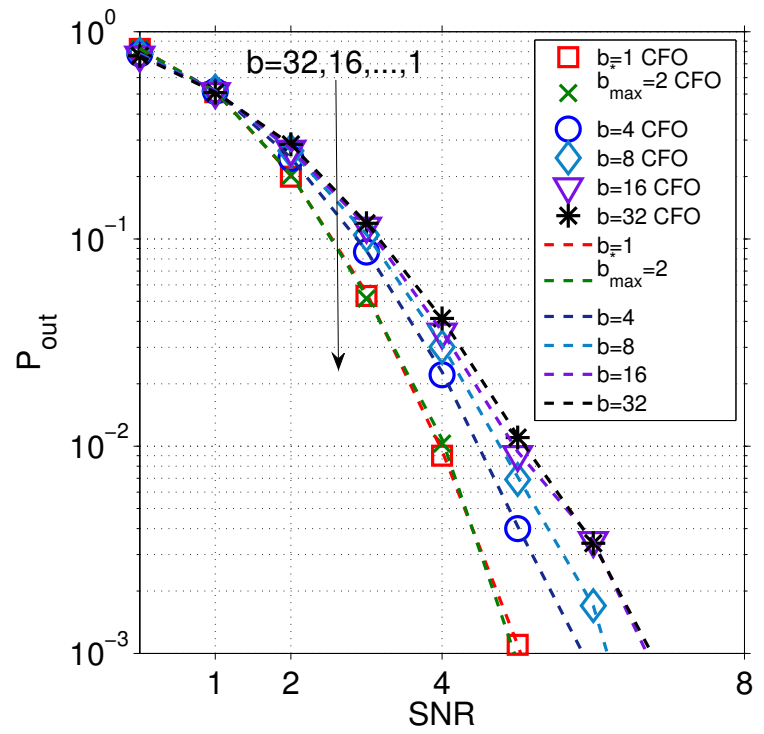

Fig. 3: Outage probability for an OFDMA system without CFO (dashed lines, appearing in decreasing order of block-size from up to down) and with CFO (marker lines) for the scenario $N_{p}=64, N_{u}=$ 2 and $L=16$. The CFO of each user is independently uniformly generated in $[0,0.01]$. The optimal block-sizes are $b^{*} \in \beta^{*}=\{1,2\}$.

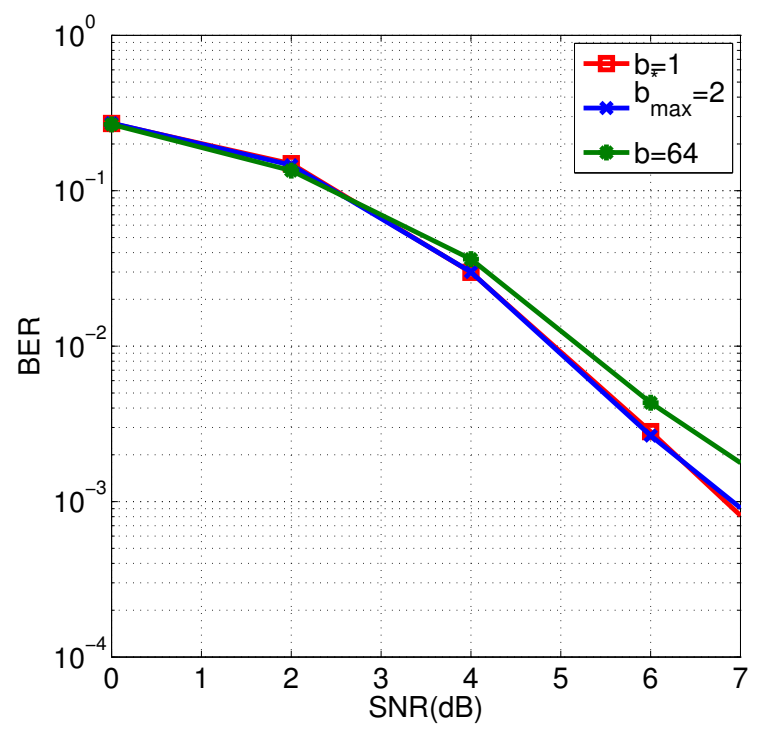

Fig. 4: Bit Error Rate for an OFDMA system with CFO for the scenario $N_{p}=128, N_{u}=2$ and $L=32$. The CFO of each user is independently uniformly generated in $[0,0.01]$. The optimal blocksizes are $b^{*} \in \beta^{*}=\{1,2\}$.

\section{CONCLusions}

This work has provided an analysis of the subcarriers block allocation policies for coded OFDMA uplink systems without channel state information and with CFO. We have extended the inter-carrier sum-correlation function given in [7] (for the case without $\mathrm{CFO}$ ) and we have provided a best block-size allocation policy minimizing the sum-correlation as trade-off

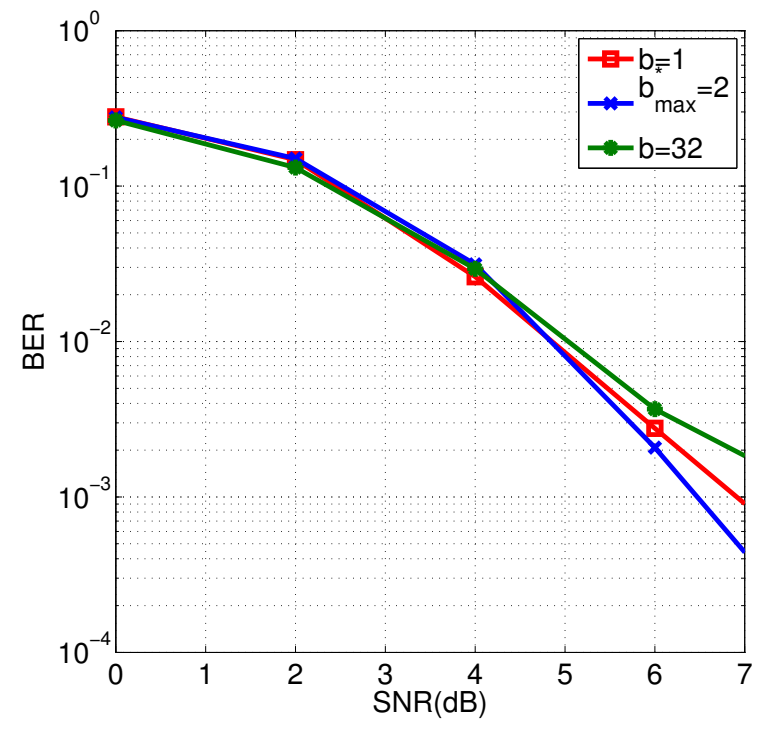

Fig. 5: Bit Error Rate for an OFDMA system with CFO for the scenario $N_{p}=128, N_{u}=2$ and $L=32$. The CFO of each user is independently uniformly generated in $[0,0.03]$. The optimal blocksize is $b_{\max }^{*}=2$.

between the diversity gain and $\mathrm{CFO}$ effects. We have also shown the robustness to CFO effects through the analysis of our proposed sum-correlation function. All these results have been validated via extensive Monte Carlo simulations.

\section{REFERENCES}

[1] A. Molisch, Wideband Wireless Digital Communication, Upper Saddle River: Prentice Hall, 2001.

[2] H. Sari, G. Karam, and I. Jeanclaude, "Channel equalization and carrier synchronization in OFDM systems," in Proceedings of the 6th Tirrenia International Workshop on Digital Communications, Tirrenia, Italy, Sep. 1993, pp. 191-202.

[3] A. Sohl and A. Klein, "Comparison of localized, interleaved, and block-interleaved FDMA in terms of pilot multiplexing and channel estimation," in Proceedings of the 15th European Signal Processing Conference, Poznan, Poland, Sep. 2007.

[4] L. Koffman and V. Roman, "Broadband wireless access solutions based on OFDM access in IEEE 802.16," IEEE Communication Magazine, pp. 96-103, Apr. 2002.

[5] B. Aziz, I. Fijalkow, and M. Ariaudo, "Trade off between frequency diversity and robustness to carrier frequency offset in uplink OFDMA system," in Proceedings of Global Telecommunications Conference (GLOBECOM 2011), Dec. 2011, Houston, Texas, pp. 1-5.

[6] S. Song, G. Chen, and K. Letaief, "Localized or interleaved? a tradeoff between diversity and CFO interference in multipath channels," IEEE Transactions on Wireless Communications, vol. 10, no. 9, pp. 2829 2834, Sep. 2011.

[7] A. Masucci, E. Belmega, , and I. Fijalkow, "Optimal blockwise subcarrier allocation policies in single carrier FDMA uplink systems," Submitted to IEEE Transactions on Signal Processing, Mar. 2013.

[8] M. Tonello and R. Bernardini, "Analysis of the achievable timefrequency diversity gains in coded OFDM," in Proceedings of the International Conference on Telecommunications, Beijing, China, Jun. 2002, pp. 5-14.

[9] L. Zheng and D. Tse, "Diversity and multiplexing: a fundamental tradeoff in multiple-antenna channels," IEEE Transactions on Information Theory, vol. 49, no. 5, pp. 1073 - 1096, May 2003.

[10] W. Yu, W. Rhee, S. Boyd, and J. Cioffi, "Iterative water-filling for gaussian vector multiple-access channels," Information Theory, IEEE Transactions on, vol. 50, no. 1, pp. 145 - 152, Jan. 2004. 